PROCEEDINGS OF THE

AMERICAN MATHEMATICAL SOCIETY

Volume 82, Number 3, July 1981

\title{
ON SUBSEQUENTIAL LIMIT POINTS OF A SEQUENCE OF ITERATES
}

\author{
M. MAITI AND A. C. BABU
}

\begin{abstract}
In this note we study the properties of the subsequential limit points of a sequence of iterates of mappings which are contractive over two consecutive elements of an orbit.
\end{abstract}

Let $A$ be a self-map of a nonempty metric space $X$. For a point $x \in X$, the sequence of iterates $\left\{A^{m} x\right\}_{m=1}^{\infty}$ may or may not converge to a point in $X$. In fixed pont theory it is an important aspect to examine convergence of the sequence of iterates or of its subsequences. It is our main aim here to study the structure of the set of subsequential limit points of a sequence of iterates.

In a recent paper Diaz and Metcalf [1] have initiated a study of the structure and properties of the set of subsequential limit points of a sequence of iterates and in doing so they have generalized a result of Tricomi [2] in connection with the iteration of a real-valued function. In this note we first make some observations on the results derived by Tricomi [2], Diaz and Metcalf [1] and show that their results may also be derived under some alternative (and possibly weaker) conditions. In fact, we have established that discontinuous mappings may also be accommodated in their works. Secondly, we arrive at the same conclusions as in [1] about the set of subsequential limit points of a sequence of iterates of mappings introduced by $\mathrm{Pal}$ and Maiti [3] and, further, this has been done under conditions weaker than those imposed by Diaz and Metcalf [1].

We now recall the following result of Tricomi [2] as established in [1].

THEOREM 1. Let $A$ be a real-valued continuous function on the (finite or infinite) open (or closed) interval $(a, b)$ whose values lies in the same interval. Suppose

(i) there exists a $p$ in $(a, b)$ such that $A p=p$,

(ii) $|A x-p|<|x-p|$ for $a<x<b, x \neq p$.

Then, for every $x$ in $(a, b), A^{m} x \rightarrow p$ as $m \rightarrow \infty$.

Our first observation on the above theorem is that the requirement of continuity of $A$ cannot be omitted. This is obvious from the following example.

EXAMPLE 1. Let $A$ be a function defined over $[-1,1]$ such that

$$
\begin{aligned}
A x & =-x / 2+1 / 2^{n+1}, & & x \in\left[-1 / 2^{n-1},-1 / 2^{n}\right), \\
& =-x / 2-1 / 2^{n+1}, \quad & & x \in\left(1 / 2^{n}, 1 / 2^{n-1}\right],
\end{aligned}
$$

Received by the editors September 27, 1979 and, in revised form, June 9, 1980.

1980 Mathematics Subject Classification. Primary 47H10; Secondary 54H25. 
for $n=1,2,3, \ldots$ and that $A 0=0$. This function maps $[-1,1]$ into itself and satisfies (i) and (ii) of Theorem 1 , but it is discontinuous at $x= \pm 1 / 2^{n}$ for $n=1,2,3, \ldots$ Further, we see that the sequence of iterates of $A$ at $x=\frac{3}{4}$, say, does not converge to 0 , the fixed point of $A$.

However, it is to be noted that the condition of continuity on the function $A$ may be weakened if we replace the condition (ii) by

(ii)' given $\varepsilon>0$ there exists a $\delta>0$ such that, for $x(\neq p) \in X, \varepsilon<|x-p|<\varepsilon$ $+\delta$ implies $|A x-p|<\varepsilon$.

It is easy to establish the conclusions of Theorem 1 with (i) and (ii)', but without the continuity of $\boldsymbol{A}$. In this connection it may be mentioned that (ii)' implies (ii), but that $A$, with (ii)', need not be continuous throughout and is continuous only at $x=p$. This is illustrated further by the following example.

Example 2. Define a function $A$ of $[-1,1]$ into itself such that

$$
\begin{aligned}
A x & =-x / 4+1 / 2^{n+1}, & & x \in\left[-1 / 2^{n-1},-1 / 2^{n}\right), \\
& =-x / 4-1 / 2^{n+1}, & & x \in\left(1 / 2^{n}, 1 / 2^{n-1}\right],
\end{aligned}
$$

for $n=1,2,3, \ldots$ and that $A 0=0$. The function $A$ satisfies (i) and (ii)' and, further, $A^{m} x \rightarrow 0$ as $m \rightarrow \infty$ for $x \in[-1,1]$. But the function is discontinuous at $x= \pm 1 / 2^{n}$ for $n=1,2,3, \ldots$.

In a similar fashion Theorem 1.1 of [1] may be modified. We now discuss the results of [1] in metric spaces. Let $A$ be a mapping of a metric space $(X, d)$ into itself. Denote the set of fixed points of $A$ in $X$ by $F(A)$. Further, for $x \in X, \mathcal{L}(x)$ will denote the set of subsequential limit points of the sequence of iterates $\left\{A^{m} x\right\}$. In this connection we state the following theorem due to Diaz and Metcalf [1] which is a generalized version of Theorem 1 .

THEOREM 2. Suppose

(i) $A: X \rightarrow X$ is continuous,

(ii) $F(A)$ is nonempty and compact,

(iii) for each $x \in X$ and $\notin F(A), d(A x, F(A))<d(x, F(A))$.

Then, for each $x \in X$, the set $\mathcal{L}(x)$ is a closed and connected subset of $F(A)$. Either $\mathcal{L}(x)$ is empty, or it contains one point, or it contains uncountably many points. In case $\mathcal{L}(x)$ is just one point, then $\lim _{m \rightarrow \infty} A^{m} x$ exists and belongs to $F(A)$. In case $\mathcal{E}(x)$ is uncountable, then it is contained in the boundary of $F(A)$.

As in the earlier case we observe here also that the continuity condition on the mapping $A$ throughout $X$ may be relaxed. Indeed, if we replace the conditions (i) and (iii) respectively by

(i) $A: X \rightarrow X$ is continuous at each point of $F(A)$,

(iii)' given $\varepsilon>0$ there exists a $\delta>0$ such that, for $x \in X$ and $\notin F(A), \varepsilon<$ $d(x, F(A))<\varepsilon+\delta$ implies $d(A x, F(A))<\varepsilon$ and keep (ii) as it is, then the same conclusions hold good except in that $\mathcal{L}(x)$ is nonempty. We only show here that $\mathcal{L}(x)$ is nonempty and the remaining conclusions may be derived following the lines of argument given in Theorem 2 of Diaz and Metcalf [1]. 
Since $F(A)$ is compact, for each iterate $A^{m} x$ there exists a $p_{m} \in F(A)$ such that $d\left(A^{m} x, F(A)\right)=d\left(A^{m} x, p_{m}\right)$. Further, from the sequence $\left\{p_{m}\right\}_{m=1}^{\infty}$ we can extract a subsequence $\left\{p_{m_{i}}\right\}_{i=1}^{\infty}$ converging to $p \in F(A)$. Also we have

$$
d\left(p, A^{m_{i}} x\right)<d\left(p, p_{m_{i}}\right)+d\left(p_{m_{i}}, A^{m_{1}} x\right) \text {. }
$$

Now, as $i \rightarrow \infty, p_{m_{i}} \rightarrow p$ and $d\left(p_{m_{i}}, A^{m_{i}} x\right) \rightarrow 0$ (this follows from (iii)'), implying $d\left(p, A^{m_{i}} x\right) \rightarrow 0$. Thus, as $i \rightarrow \infty, A^{m_{i}} x \rightarrow p \in \mathcal{L}(x)$ and hence $\mathcal{L}(x)$ is nonempty.

Next, we show that the conditions (ii) and (iii) of Theorem 2 imply the closure of the orbit $O(x, A)$ of a point $x \in X$ to be compact, i.e. $\bar{O}(x, A)$ is compact. Obviously, $\bar{O}(x, A)=O(x, A) \cup \mathcal{L}(x)$. Consider a sequence $\left\{y_{n}\right\}_{n=1}^{\infty} \subset \bar{O}(x, A)$. If $\left\{y_{n}\right\}_{n=1}^{\infty}$ contains an infinite number of points from $\mathcal{L}(x)$, then from the compactness of $\mathcal{L}(x)$ it follows that $\left\{y_{n}\right\}_{n=1}^{\infty}$ contains a convergent subsequence. If $\left\{y_{n}\right\}_{n=1}^{\infty}$ contains a finite number of points from $\mathcal{L}(x)$, then there exists a $k$ such that $y_{n} \in O(x, A)$ for $n \geqslant k$. Since $F(A)$ is compact, there exists a $p_{n}$ such that $d\left(y_{n}, F(A)\right)=d\left(y_{n}, p_{n}\right)$ for $n \geqslant k$. Now $\left\{p_{n}\right\}_{n=k}^{\infty}$ contains an infinite subsequence $\left\{p_{n_{i}}\right\}_{i=1}^{\infty}\left(n_{i}>k\right)$ converging to $p \in F(A)$. Then

$$
d\left(p, y_{n_{i}}\right) \leqslant d\left(p, p_{n_{i}}\right)+d\left(p_{n_{i}}, y_{n_{i}}\right) \rightarrow 0 \text { as } i \rightarrow \infty
$$

implying $y_{n_{i}} \rightarrow p \in \mathcal{L}(x)$. Thus, whatever may be the case, the sequence $\left\{y_{n}\right\}_{n=1}^{\infty} \subset$ $\bar{O}(x, A)$ always has a subsequence converging to a point $p \in \bar{O}(x, A)$. This shows that $\bar{O}(x, A)$ is compact.

We now use the compactness of $\bar{O}(x, A)$ in the following theorem and derive the same conclusions as those of Diaz and Metcalf [1] in the case of the mappings introduced by Pal and Maiti [3, Theorem 2].

TheOREM 3. Let $A: X \rightarrow X$ be a continuous mapping and $O(x, A)$ be the orbit of $a$ point $x \in X$. Suppose that $\bar{O}(x, A)$ is compact and $A$ satisfies, for any two distinct elements $x, y \in X$, at least one of the following conditions:

(i) $d(x, A x)+d(y, A y)<2 d(x, y)$,

(ii) $d(x, A x)+d(y, A y)<\frac{2}{3}\{d(x, A y)+d(y, A x)+d(x, y)\}$,

(iii) $d(x, A x)+d(y, A y)+d(A x, A y)<\frac{3}{2}\{d(x, A y)+d(y, A x)\}$,

(iv) $d(A x, A y)<\max \left\{d(x, y), d(x, A x), d(y, A y), \frac{1}{2}[d(x, A y)+d(y, A x)]\right\}$.

Then $\mathcal{L}(x)$ is a nonempty, closed and connected subset of $F(A)$. Either $\mathcal{L}(x)$ contains exactly one point or uncountably many points. In case $\mathcal{L}(x)$ is a singleton, $\lim _{m \rightarrow \infty} A^{m} x$ exists and belongs to $F(A)$. In case $\mathcal{L}(x)$ is uncountable, it is contained in the boundary of $F(A)$.

Proof. It has been shown in [3] that the compactness of $\bar{O}(x, A)$ ensures the existence of subsequential limit points, which are also fixed points of $A$. Thus $\mathcal{L}(x)$ is never empty in this case. Then $\mathcal{L}(x)$ is a closed subset of $F(A)$, which, because of the continuity of $A$, is also closed.

To prove the connectedness of the set $\mathscr{L}(x)$ we assume the contrary, i.e. let $\mathcal{L}(x)=S_{1} \cup S_{2}$, where $S_{1}$ and $S_{2}$ are both nonempty, closed and disjoint. Since $\mathcal{L}(x)$ is a closed subset of the compact set $\bar{O}(x, A)$, then $\mathcal{L}(x)$ is also compact. Further, $S_{1}$ and $S_{2}$, being closed subsets of $\mathcal{L}(x)$, are also compact and hence 
$d\left(S_{1}, S_{2}\right)>0$. It has also been shown in [3] that the mapping $A$ is asymptotically regular, i.e. $d\left(A^{m} x, A^{m+1} x\right) \rightarrow 0$ as $m \rightarrow \infty$.

We now need to show that $d\left(A^{m} x, \mathcal{L}(x)\right) \rightarrow 0$ as $m \rightarrow \infty$. To do so we assume the contrary. Then there exist an $\varepsilon>0$ and a sequence of integers $\left\{m_{i}\right\}_{i-1}^{\infty}$ such that $d\left(A^{m_{i}} x, \mathcal{L}(x)\right)>\varepsilon>0$. Since $\bar{O}(x, A)$ is compact, then the sequence $\left\{A^{m_{1}} x\right\}_{i=1}^{\infty}$ has a subsequence, say $\left\{A^{n_{i}} x\right\}_{i=1}^{\infty}$, converging to $p \in \mathcal{L}(x)$. Therefore $d\left(A^{m^{m}} x, p\right)<\varepsilon$ for large values of $i$, implying a contradiction.

From the above results we see that there exists an integer $M$ such that for $m>M$

$$
\begin{aligned}
& d\left(A^{m} x, A^{m+1} x\right)<\frac{1}{3} d\left(S_{1}, S_{2}\right), \\
& d\left(A^{m} x, S_{1} \cup S_{2}\right)=d\left(A^{m} x, \mathcal{L}(x)\right)<\frac{1}{3} d\left(S_{1}, S_{2}\right) .
\end{aligned}
$$

Since $\mathcal{L}(x)=S_{1} \cup S_{2}$ is compact, there exists a $\mathcal{S} \in S_{1} \cup S_{2}$ such that

$$
d\left(A^{m} x, S_{1} \cup S_{2}\right)=d\left(A^{m} x, \delta\right) \text {. }
$$

If $\mathcal{S} \in S_{1}$, then $d\left(A^{m} x, S_{1}\right)<d\left(A^{m} x, \delta\right)<\frac{1}{3} d\left(S_{1}, S_{2}\right)$. Therefore, for any $m \geqslant M$, either

$$
d\left(A^{m} x, S_{1}\right)<\frac{1}{3} d\left(S_{1}, S_{2}\right)
$$

or

$$
d\left(A^{m} x, S_{2}\right)<\frac{1}{3} d\left(S_{1}, S_{2}\right) .
$$

But both these inequalities cannot hold simultaneously for the same $m$ because in that case

$$
d\left(S_{1}, S_{2}\right) \leqslant d\left(A^{m} x, S_{1}\right)+d\left(A^{m} x, S_{2}\right)<\frac{2}{3} d\left(S_{1}, S_{2}\right),
$$

which is absurd.

Next, we claim that the set of positive integers $m>M$ satisfying $d\left(A^{m} x, S_{1}\right)$ $<\frac{1}{3} d\left(S_{1}, S_{2}\right)$ is nonempty, since $S_{1} \subset \mathcal{L}(x)$ and $S_{1}$ is nonempty. Similarly, the set of positive integers $m>M$ for which $d\left(A^{m} x, S_{2}\right)<\frac{1}{3} d\left(S_{1}, S_{2}\right)$ is nonempty. Suppose that, for $m_{1}>M, d\left(A^{m_{1}} x, S_{1}\right)<\frac{1}{3} d\left(S_{1}, S_{2}\right)$. Then there exist integers $n>m_{1}$ such that $d\left(A^{n} x, S_{2}\right)<\frac{1}{3} d\left(S_{1}, S_{2}\right)$. Let $k+1$ be the smallest of such integers, and we have

$$
d\left(A^{k} x, S_{1}\right)<\frac{1}{3} d\left(S_{1}, S_{2}\right)
$$

and

$$
d\left(A^{k+1} x, S_{2}\right)<\frac{1}{3} d\left(S_{1}, S_{2}\right)
$$

Then one has

$$
\begin{aligned}
d\left(S_{1}, S_{2}\right) & <d\left(S_{1}, A^{k} x\right)+d\left(A^{k} x, A^{k+1} x\right)+d\left(A^{k+1} x, S_{2}\right) \\
& <\frac{1}{3} d\left(S_{1}, S_{2}\right)+\frac{1}{3} d\left(S_{1}, S_{2}\right)+\frac{1}{3}\left(S_{1}, S_{2}\right)=d\left(S_{1}, S_{2}\right)
\end{aligned}
$$

which is absurd. Therefore the hypothesis that $\mathcal{L}(x)=S_{1} \cup S_{2}$, where $S_{1}$ and $S_{2}$ are nonempty, closed and disjoint, is wrong. Hence $\mathcal{L}(x)$ is connected. 
The fact that $\mathcal{L}(x)$ is either a singleton or uncountable follows readily from Theorem 1 of Berge [4, p. 96]. In case $\mathcal{L}(x)$ is a singleton $\{p\}, d\left(A^{m} x, p\right)=$ $d\left(A^{m} x, \mathcal{L}(x)\right) \rightarrow 0$ as $m \rightarrow \infty$. Following the same arguments as those of Diaz and Metcalf [1] it is possible to show that $\mathcal{L}(x)$ is contained in the boundary of $F(A)$, in case it is uncountable.

REMARK 1. In the above theorem the continuity condition on $A$ may be removed if $A$ satisfies the conditions (i) and (ii) only. Further, if $A$ satisfies (iv) only, then $\mathcal{L}(x)$ is just one point.

Remark 2. If, instead of $A$, the $k$ th iterate $A^{k}$ is continuous, then with $A^{k}$ a corresponding theorem may be formulated resulting in the same conclusions as those in Theorem $2^{k}$ of [1].

ACKNOWLEdGement. The authors wish to thank the referee for pointing out mistakes in the original version of the paper.

\section{REFERENCES}

1. J. B. Diaz and F. T. Metcalf, On the set of subsequential limit points of successive approximations, Trans. Amer. Math. Soc. 135 (1969), 459-485.

2. F. Tricomi, Un teorema sulla convergenza delle successioni formate delle successive iterate di una funzione di una variabile reale, Giorn. Mat. Battaglini 54 (1916), 1-9.

3. T. K. Pal and M. Maiti, Extensions of fixed point theorems of Rhoades and Ciric, Proc. Amer. Math. Soc. 64 (1977), 283-286.

4. C. Berge, Topological spaces, Oliver \& Boyd, London, 1963.

Department of Mathematics, Indin Institute of Technology, Kharagpur, India 